\title{
Framework for automatic creation of remote operations support systems
}

\author{
J.P.T. Mo and L. Nemes \\ CSIRO Manufacturing Science and Technology, Corner of \\ Albert and Raglan Streets, Preston, Vic. 3072, Australia. \\ Ph: +6139662 775, Fax: +61396627851 \\ Em:jmo@cmst.csiro.au
}

\begin{abstract}
Customer service is essential to maintain good and lasting relationships. Many companies would like to reduce the cost of such support services. They have been looking for solutions many of which are created using IT tools. This paper will discuss a generic approach using IDEF3 process modelling methodology to provide such services in a flexible fashion. The approach results in the definition of a framework consisting of generic modelling methodology and software systems which captures the knowledge of experts and generates customer support systems tailored to the application requirements. The framework allows personnel, who do not have computer programming background, to generate such systems easily and the information can be accessed anywhere in the world.
\end{abstract}

\section{Keywords}

Remote customer support, Process modelling, WWW technologies, Knowledge based system, IDEF3, HTML structure

\section{INTRODUCTION}

As the world's economy becomes global, manufacturing operations are facing new challenges to improve their quality of the products and services within the complete product life cycle (Lee, 1994). The strategy to extend customer support 
from the usual design oriented supplier's view to a comprehensive coverage beyond the sale and guarantee period of the product has emerged among manufacturers who operate globally and produce large equipment with typical life span of over 20 years. Manufacturers have to advise their customers on various aspects of their products and give guidance to on how their products can be operated, and when and how to do preventive maintenance. They also have to help customers on how to identify the causes for eventual malfunctions and how to repair them if the equipment fails. All these are imposing serious challenges to the manufacturer because the interactions with customers have to be done promptly and efficiently.

Another issue which requires extensive research is how the support information should be organised for different kinds of people. A novice machine operator requires more detailed instructions than a trained one. People in different countries have been brought up in distinct traditions and education systems (Robin, 1996). Their communication is hindered not only by language difficulties but also by cultural differences. The system should therefore be flexible and adaptable to the needs of the user irrespective of the differences involved.

In the Globeman 21 project, which is part of the Intelligent Manufacturing Systems (IMS) international program, we look at the processes which are envisaged to be essential for manufacturing industry in the coming years. This is particularly important for products, which are investment in nature and whose operation is generally sophisticated, for example, the CNC machines domain.

This paper describes an industry-led project to investigate a novel framework which integrates a number of technologies to provide after sales services to customers. Vendors can use the framework to build models which describe their desired patterns of remote operations support and subsequently generate the system which their customers will use. Experience in the application of this framework to a CNC machine manufacturer has achieved substantial savings in creating and maintaining the remote customer operations support system.

The importance of the product (e.g. CNC machine) life cycle aspect could be seen from the revenue generated by maintenance and renewal business for the vendor. Traditionally, manufacturers concentrated their efforts in research and development to create the product which is superior in "cost to performance" to those of the competitors. The activities from the delivery of the product to the end of its life cycle are provided to an extent barely sufficient to keep the product operational for the satisfaction of the customers. The change in global market forces vendors to rethink about this philosophy. More and more vendors realize that provision of services related to the use and maintenance of the equipment is a tremendous opportunity to expand their business. A shipbuilding company estimated that the business generated after the sale till dismantling the ship, could be twice as much as the sale price of the product. Understanding such technical and economic impact and its technical components are therefore paramount for companies thinking on a global scale. 
Similarly, the same concept can be applied to companies which design, manufacture and support large installations of their own. Their customers are in fact internal. They include operators, line managers and company management. The operational activities are those after the installation of the equipment and will be supported by the servicing team. Well organized operational and servicing support not only helps to ensure high efficiency and quality of the output, it also extends the usable life of the equipment so that more profits can be made.

\section{CHARACTERISTICS OF REMOTE OPERATIONS SUPPORT}

A significant difference in the characteristics of remote operations support services as compared to those activities prior to sales is that they often occur outside of the vendor company's physical boundaries. In many cases, these services are provided remotely, that is, at a substantial distance from the vendor. A review of literature shows that most remote support services are concentrated on diagnosis of machines or the problems in the manufacturing processes. For example, control systems manufacturers have diagnosed faulty boards through telephone networks and ordered replacement parts (Huang and Wang, 1996). Diagnosis and support for marine operations can be done via satellite connections (Karsal et al, 1996). A good communication network and commonality of formats are essential requirements in both cases.

On the other hand, the ability of the system to provide a good answer to the customer must be considered. Artificial intelligence techniques such as expert systems (Kim et al, 1996), neural networks (Chen et al, 1996) and many others (Patel and Kamrani, 1996, Chatwin et al, 1996) have been employed. Obviously, the final implementation of the remote operations support has to be integrated and the answers unified.

\section{SERVICES IN DEMAND}

In the one-of-a-kind product life cycle, many kinds of operations support services can be identified. The research in CSIRO has concentrated in four particular types, namely, Remote Diagnostics, Remote Expert Advice and Process Optimization, Remote Training and Remote On-line Manual. The outcomes from the research are integrated by the Framework for Integration of Remote Support Technologies (FIRST).

\section{Remote Diagnostics}

In Remote Diagnostics support, there are many non-controllable factors involved in the process, for example, changes in the conditions of the machine itself or the input materials. For these well understood processes, the ability to carry out 
diagnostics remotely is less critical. However, for products built on less disciplined type of knowledge such as CNC operations, these factors are normally unpredictable, although in many cases are repeatable. However, the major problem with these approaches is that each of these remote diagnostics system normally involves experts from scratch. This proves to be expensive because they are one-off in nature. Experience also shows that knowledge gained in one project is not formally recorded and hence is difficult to be transferred to other projects.

\section{Remote Expert Advice and Process Optimization}

Expert Advice refers to the support service which provides advice to the user on what operating parameters can be used to achieve best results. It involves the search of a knowledge base which consists of engineering design knowledge, expert knowledge in scientific investigations, past operating results and rules to evaluate.

In addition to field knowledge, there is also a need to incorporate new knowledge into the system as the technology develops. Existing systems are generally robust in this sense because they are often designed with a particular problem in mind and hence are deficient in the flexibility to accommodate structural changes of the knowledge representation. Substantial effort is then required to integrate the existing knowledge base with the new knowledge (Aamodt and Nyard, 1995). The changes in knowledge structure also impose problems in the delivery of the support to the customer.

\section{Remote Training and On-line Manual}

In order to maintain consistent attention from the trainees and system users, multimedia authoring tools are now available to create applications which can be used for self-training or as an on-line manual (Asymetrix Corp., 1995). These tools normally run on a PC and so are excellent systems for localized support. However, for customers and users who are far away, some other form of delivery has to be employed. For example, graphics information has been successfully displayed by hypertext technology for a local support situation (Koshy et al, 1996).

\section{FORMAL MODELLING}

In general, the current approach to develop a remote operational support system is a four step process as shown in Figure 1. Initially, the expert of the machine process defines the operational topics to be supported. The description is then passed to a system analyst who will design the system to handle the requirements specified by the expert. This will take many rounds of discussions before the final format of the knowledge can be represented properly. Programmers are then made 
available to the project to transform this idea to practices. When the system is completed, the result is released to the user.

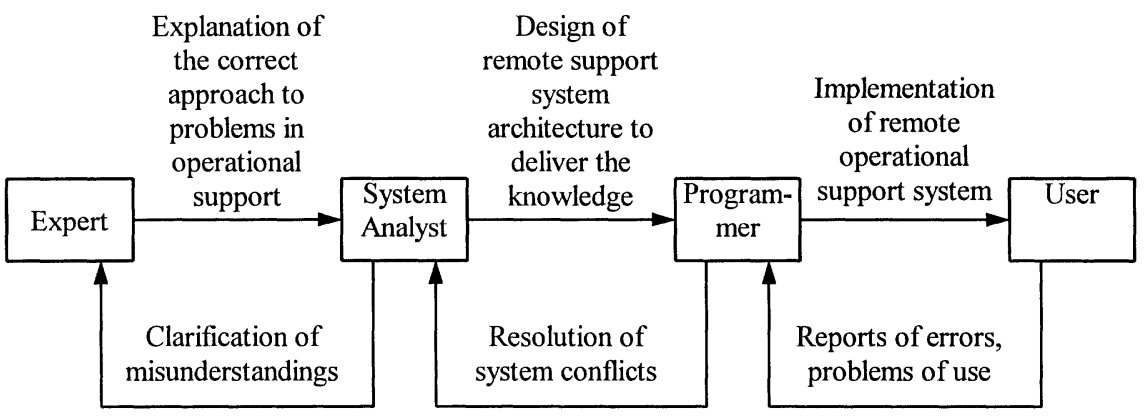

Figure 1 Current process of remote operational support systems development.

This approach suffers from the fact that each of the remote operations support systems are created as an individual developmental project. This is a time consuming and expensive process. The results are often simply a repetition of many basic components of some other applications but with specific adaptation to the new requirements. These repeated developments can well be picked up by the system automatically from previous projects so that the process of system development can be more productive.

Moreover, the expert in Figure 1 does not have a direct relationship with the final remote operations support system. Hence, the person who develops the system is not the person who has the most relevant experience. Current tools are designed for system analysts and programmers. They are unable to handle cognitive demand to represent the knowledge the expert provides in a structured way so that such knowledge can be transformed into a remote operational system.

We need a generic approach by which people who have experience can work on the information they want to deliver. In general, human knowledge is not well structured and often relies on the understanding of individuals. In order to enable better information transfer, a formal modelling approach provides a structure means of achieving these goals and simplifies the complexity of communication (Barkan et al, 1993). In our research, we regard the remote customer support as a process which can be described conveniently by an IDEF3 model as shown in Figure 2. IDEF stands for ICAM DEFinition methods. It results from a series of research carried out by the US Air Force in 1970s (Bravoco and Yadav, 1985). IDEF3 is one of the methods developed specifically for describing processes (KBSI, 1997).

The remote customer support process is in fact a loop consisting of a series of dialogues with the remote customer. The system works with the knowledge base to provide answers according to the feedback from the customer. 


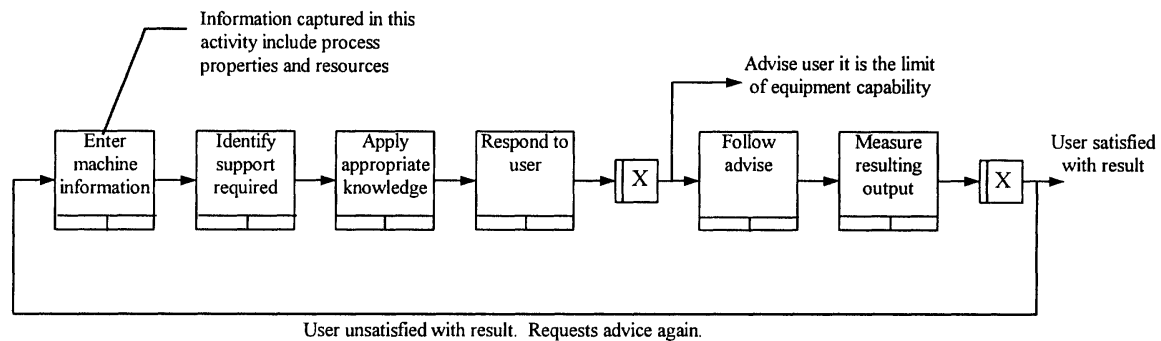

Figure 2 Current remote operational support processes.

\section{THE SYSTEM FRAMEWORK}

The approach provides integration of the technologies: World Wide Web (WWW), hypertext markup language (HTML), interactive forms, graphics, video/audio, artificial intelligence, supported by IDEF3 modelling methodology, object oriented technology, Java. The system consists of 4 blocks as shown Figure 3.

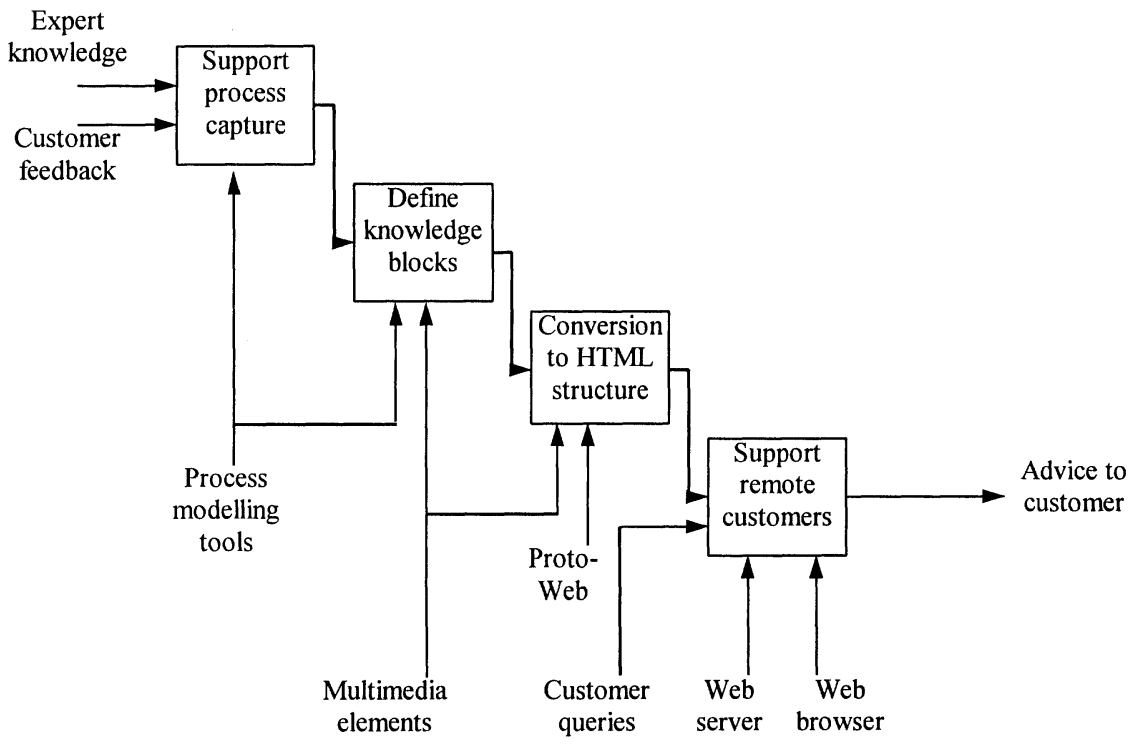

Figure 3 System framework.

The essence of the approach is to use IDEF3 process modelling tools to capture the knowledge of the expert in an explicit fashion. The logistics and procedures of solving problems for customers are represented as a series of process 
charts which can be easily understood by any personnel. Knowledge blocks are defined which include problem solvers that are not divisible but can be called up to provide the answers easily. Once the model is captured, the modelling tools will be used to convert the model into a readable format so that it can be further converted into the final deliverable.

IDEF3 is an object-oriented process modelling language. Object oriented methods have proved very useful in many manufacturing system development (Basnet and Mize, 1995). In IDEF3, a process block is defined in similar way as a class in a computer language. When it comes to the point of inserting the process into an activity chain, the process block is instantiated. Hence, the same process can appear at different steps of the whole cycle. Moreover, attributes which are defined from the generic process block are inherited. For example, the process description, facts and constraints are automatically picked up during the instantiation procedure. A lot of time can then be saved due to repeated use of objects.

In order to enable an interactive process model browsing and manipulation, a Java program is incorporated as an applet into every HTML page. The applet is activated by a "WEB MAP" button conveniently located on each page and activates the Java program on a separate window. A "model description file" is written by the conversion program and downloaded as part of the applet definition on the HTML page. The applet is downloaded and run without entry sound play but messages can be seen at the bottom of the Web browser. The blocks in the applet are active. If the pointer is inside the button boundary, the URL for that block is automatically displayed at the dialogue section of the applet window. Clicking the block will activate the Web page.

In order to attract the user in the interface, the registration block where the user is reading is displayed in pink. Blocks which has further decomposition are coloured dark grey with an extended flag to indicate that more model blocks are expected at lower levels from there. Other blocks are displayed as light grey. Other functions such as controlled URL in the applet are also included.

When the HTML structure is generated from an IDEF3 model, it is imperative to ensure that it knows where to find the other components of the system. In FIRST, this convention is handled by a pre-defined directory structure which takes care of general and individual company's needs.

The directory structure to hold the files is designed with a relative designation from a starting point defined by the user in his/her home page. The user can then create a URL from the home page to the Web pages generated by FIRST as shown in Figure 4. 


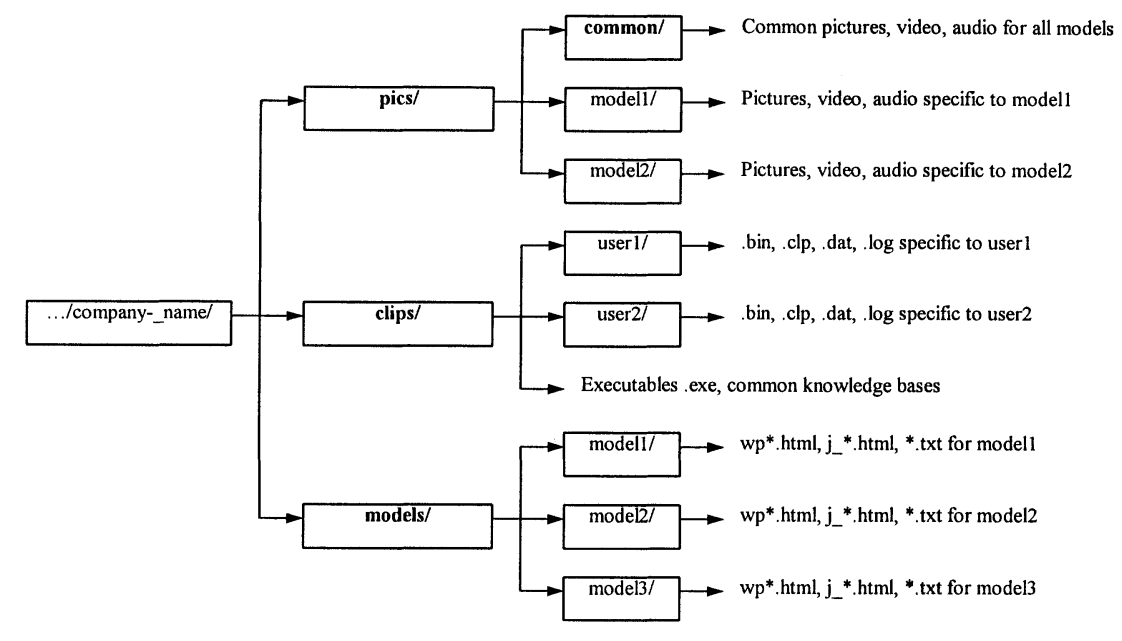

- Bold types are the actual directory name

- Normal types are changed according to requirements

Figure 4 Directory structure to support FIRST.

The pics/ directory has a common/ sub-directory and models/ sub-directories. All pictures, video, audio files are stored in this directory. The common/ subdirectory contains pictures, video and audio files which are common to all models. A typical example is the vacation.gif file which is included in all models to indicate the job is done.

The models/ sub-directories contain similar types of files but are specifically used for that model only. If the model is removed from the server, the relevant sub-directory will be removed too.

The clips/ directory contains all the executables which are activated by CGIbin scripts. It also contains the common knowledge bases for all users. For knowledge and information specific to individual users, user sub-directories are created under clips/ and contain data files which will be read in by the executables. They are the working directories of each user session. Examples of the data files include .bin, .clp, .dat, .log files. Some of these files are created by the executables during run time. This arrangement separates the data files from individual users so that information can be specific to the need of individual customers.

\section{AN IMPLEMENTATION EXAMPLE}

This paper describes an industry project involving a CNC machine manufacturer. The industry partner has customers all over the world and hence needs to provide effective and responsive remote support to customers in the use, maintenance and troubleshooting of their equipment. As the market for CNC machines expands, 
these needs will increase extensively in the next few years so that the current support processes will no longer be economically viable. In addition, the company desires to capture the vast expert knowledge within and outside the company concerning their $\mathrm{CNC}$ machines, especially as it pertains to problem diagnosis. However, there is no ready means of gathering and storing this knowledge, and hence no way to exploit it for remote customer support.

From these observations, the framework for creating the remote customer support system is built around a knowledge repository consisting of field and engineering knowledge of the industry partner and their customers, as shown in the system architecture in Figure 5. Four user interfaces, Remote Machine Diagnostics, Remote Process Optimisation, Remote Knowledge Acquisition and Remote Operator Training, which interact with the user at a remote location, interrogate the Knowledge Repository to provide appropriate information to the user. In this way, all functions can be kept up-to-date easily through the latest network technologies.

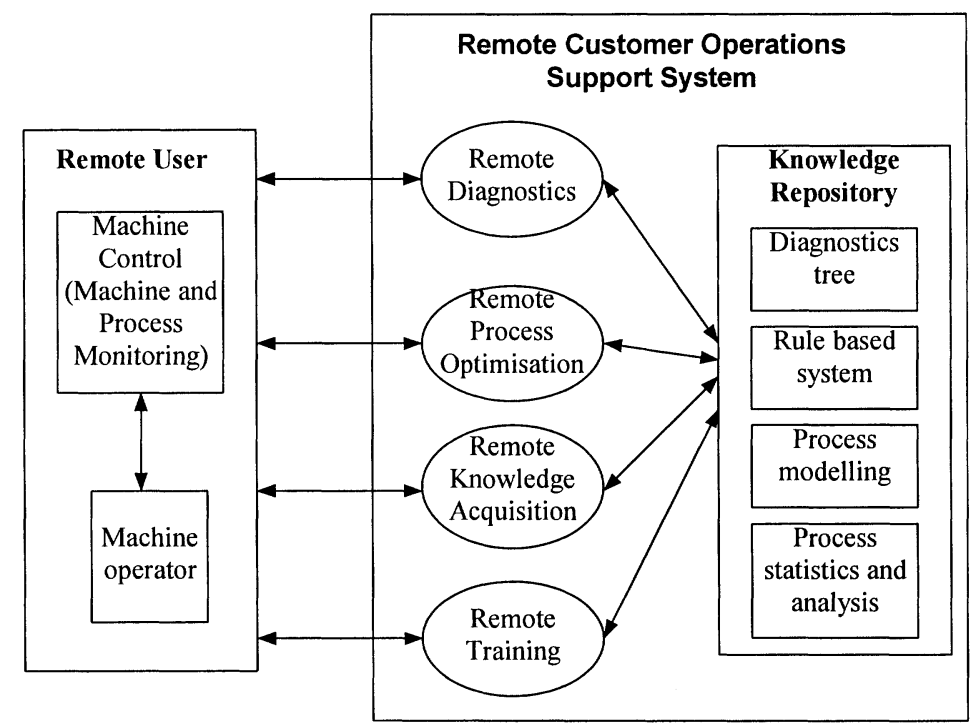

Figure 5 Current process of remote operational support systems.

The creation of the system started from the IDEF3 model screen as shown in Figure 3. Process blocks are defined using the modelling software so that the creator can have a true "MAP" of the customer support process. 
A framework has been presented in this paper to address the issue of flexibility and effective remote customer operations support. The approach starts with the use of IDEF3 process modelling methodology to describe the series of activities that a remote operator may interact with the expert engineers back in the supplier company. The model is created using a commercial IDEF3 software which provides easy manipulation of model and user friendliness. The resulting model is then transformed into a structure of HTML pages by a conversion program developed by CSIRO.

The essence of this approach is to eliminate the difficulties that normal service and support engineers need to handle to create systems in complex operations and service knowledge. The framework provides methods of defining the elements, including multimedia components into the model and subsequently generated automatically. This characteristics simplifies the work of engineers significantly and hence helps engineers to develop systems which are more relevant to the customer's needs.

The framework has been applied to allow engineers of a CNC manufacturer to generate their own supporting information system. Further development of the system has been planned within the Globeman 21 partner's community. It is anticipated that the system will be bundled with the CNC manufacturer's product in the future.

\section{REFERENCES}

Aamodt A., Nygard M. (1995). Different Roles and Mutual Dependencies of Data, Information, and Knowledge - An AI Perspective on Their Integration, Data and Knowledge Engineering, 16, 191-222

Asymetrix Corporation (1995). Toolbook User Manual and Ver.4 Update

Barkan P., Hinckley C.M. (1993). The Benefits and Limitations of Structured Design Methodologies, Manufacturing Review, 6(3), September, 211-20

Basnet C., Mize J.H. (1995). A Rule-based, Object-oriented Framework for Operating Flexible Manufacturing Systems, Int.J.Prod.Res., 33(5), 1417-31

Bravoco R.R., Yadav S.B. (1985). Requirement Definition Architecture - An Overview, Computers in Industry, 6, 237-51

Chatwin C.R., Abdullah H.A., Watson I.A. (1996). A Knowledge-based System for Operations Management in a Small to Medium Sized Enterprise, The International Journal of Advanced Manufacturing Technology, 11, 381-6

Chen Y.B., Li X., Orady E. (1996). Integrated Dignosis Using Information-GainWeighted Radial Basis Function Neural Networks, Computers and Industrial Engineering, 30(2), 243-55 
Huang H.H., Wang H.P.B. (1996). Machine Fault Diagnostics Using a Transputer

Network, Computers and Industrial Engineering, 30(2), 269-81

Karsal G., Padalkar S., Franke H., Sztipanovits J., DeCaria F. (1996). A Practical Method for Creating Plant Diagnostics Applications, Integrated ComputerAided Engineering, 3(4), 291-304

KBSI (Knowledge Based Systems, Inc.) (1997). Introduction to IDEF, http://www.kbsi.com/idef/

Kim K.Y., Kang M.S., Song G.W., Kim C.T. (1996). Development of Maintenance Supporting Expert System for Thermal Power Plant Boilers", Proceedings of the Pacific Conference on Manufacturing Engineering, 1, 2931 October, Seoul, Korea, 78-83

Koshy T.T., Gramopadhye A.K., Kennedy W.J., Ramu N.V. (1996). Application of Hypertext Technology to Assist Maintenance on the Shop Floor, Computers and Industrial Engineering, 30(2), 283-95

Lee J (1994). Manufacturing Globalization: New Challenges for the ComputerIntegrated Manufacturing, Proceedings of the $4^{\text {th }}$ International Conference on Computer Integration Manufacturing and Automation Technology, 10-12 October, Troy, U.S.A., 206- 7

Patel S.A., Kamrani A.K.K. (1996). Intelligent Decision Support System for Diagnosis and Maintenance of Automated Systems, Computers and Industrial Engineering, 30(2), 293-319

Rubin S.H. (1996). Computer-assisted Instruction in Engineering Education and Training, Journal of Computers and Industrial Engineering, 30(4), 765-79

\section{BIOGRAPHY}

John Mo received his BSc in Mechanical Engineering and MSc in Industrial Engineering from the University of Hong Kong, and his $\mathrm{PhD}$ from Loughborough University of Technology, UK. He has 23 years of experience in industry and universities in various capacities including principal lecturer, laboratory manager and senior engineer. His research interests are in concurrent engineering, systems analysis and integration, process planning, real-time control and automation. He is a corporate member of a number of professional institutions and currently project manager at CSIRO.

Dr Laszlo Nemes is a Chief Research Scientist and Program Leader in CSIRO Manufacturing Science and Technology. He has led complex research teams to complete large, industry related $\mathrm{R} \& \mathrm{D}$ projects in the area of computer aided design and manufacturing. His achievements are hallmarked with six industry related patents, 92 papers published internationally, six books/monographs. His recent achievement, the development of the Generic Enterprise Reference Architecture, has further enhanced his world wide reputation. 